\title{
Convergence diffuse d'une suite de fonctions
}

par

\section{Jean Saint Raymond (Paris)}

Abstract. The result we prove here is somewhat stronger than the following: "Let $\left(f_{n}\right)$ be a sequence of continuous functions on $[0,1]$. Then the sequence of integrals $\left(\int f_{n}(t) d \sigma(t)\right)$ converges for every atomless measure $\sigma$ on $[0,1]$ if and only if the sequence $\left(f_{n}\right)$ is uniformly bounded and every subsequence from $\left(f_{n}\right)$ contains a pointwise converging subsequence".

Le point de départ de cette étude est la constatation que la suite des fonctions de Rademacher sur $[0,1]$, bien qu'elle converge faiblement vers 0 dans l'espace $L^{\mathbf{1}}$ associé à la mesure de Lebesgue, ne converge pas faiblement vers 0 dans l'espace $L^{1}$ associé à n'importe quelle mesure diffuse.

On a donc cherchéè̀ quelles conditions une suite $\left(f_{n}\right)$ de fonctions continues sur un compact $K$ converge faiblement vers 0 dans $L^{1}(\sigma)$ pour toute mesure diffuse $\sigma$ sur $K$, ou ce qui revient au même, à quelles conditions $\left(\int f_{n} d \sigma\right)$ converge vers 0 pour toute probabilité diffuse $\sigma$. Le résultat essentiel est alors qu'une sous-suite de $\left(f_{n}\right)$ converge vers 0 en dehors d'un ensemble dénombrable.

Plus généralement, on considère dans toute la suite un compact métrisable $K$ et une face $F$ du convexe des mesures de probabilité $\mathscr{M}^{1}(K)$ sur $K$, dont on suppose qu'elle est un $G_{\delta}$ pour la topologie vague, et on cherche des conditions nécessaires et suffisantes sur une suite $\left(f_{n}\right)$ de fonctions continues sur $K$ pour que $\int f_{n} d \sigma$ converge pour tout $\sigma$ de $F$.

Definition 1. On dira qu'une partie $A$ de $K$ est $F$-négligeable si elle est négligeable pour toute mesure $\sigma$ de $F$. On notera $\mathcal{N}$ la famille des boréliens $F$-négligeables de $K$.

Definition 2. On appelle support de $F$ le complémentaire du plus grand ouvert $F$-négligeable, c'est-à-dire le plus petit fermé portant toutes les mesures de $F$.

Dans le cas où $F$ est la famille des mesures de probabilité diffuses sur $K$, qui est une face vaguement $G_{\delta}$, le support de $F$ est le noyau parfait de $K$, et les boréliens $F$-négligeables sont les parties dénombrables de $K$.

La démonstration suivante est laissée au lecteur.

LEMME 3. La face $F$ s'identifie à une face vaguement $G_{\delta}$ dense de $\mathscr{M}^{1}\left(K_{0}\right)$, où $K_{0}$ est le support de $F$ 
On peut ainsi, en considérant les restrictions à $K_{0}$ des fonctions $\left(f_{n}\right)$ se ramener au cas où $F$ est vaguement $G_{\delta}$ dense dans $\mathscr{M}^{1}(K)$.

LEMME 4. L'espace vectoriel $V$ engendré par $F$ est normiquement fermé dans l'espace $\mathscr{M}(K)$ des mesures de Radon sur $K$.

Démonstration. Puisque $F$ est une face de $\mathscr{M}^{1}(K)$, la trace de $V$ sur le cône $\mathscr{M}(K)$ des mesures positives sur $K$ est le cône engendré par $F$. Puisque $F$ est vaguement' $G_{\delta}, F$ est un $G_{\delta}$ normique.

L'application $\mu \rightarrow \mu /\|\mu\|$ est normiquement continue sur $\mathscr{M}(K) \backslash\{0\}$. Donc

$$
V \cap \mathscr{M}^{+}(K)-\{0\}=\left\{\mu \mid \frac{\mu^{\circ}}{\|\mu\|} \in F\right\}
$$

est un $G_{\delta}$ normique dans $\mathscr{M}(K) \backslash\{0\}$, de même que $V \cap \mathscr{M}^{+}(K)$ dans ${ }^{\cdot} \mathscr{M}(K)$ Puisque $V$ est l'ensemble des mesures telles que $\mu^{+}$et $\mu^{-}$soient dans $V \cap \mathscr{M}^{+}(K)$, et que les applications $\mu \rightarrow \mu^{+}$et $\mu \rightarrow \mu^{-}$sont normiquement continues, $V$ est un $G_{\delta}$ pour la norme. Mais comme $V$ est un sous-espace vectoriel de l'espace de Banach $\mathscr{M}(K)$, ceci entraîne que $V$ est fermé en norme, d'après le théorème de Baire.

Definition 5. On appellera topologie diffuse sur $C(K)$ la topologie faible associée à la dualité avec l'espace $V$ engendré par $F$. On dira qu'une suite $\left(f_{n}\right)$ converge diffusément si elle converge pour cette topologie; bien sûr cette convergence dépend de $F$, qu'on a supposé fixé une fois pour toute.

Cețte topologie est séparée si et seulement si $K$ est le support de $F$.

Proposition 6. Si $Z$ est une partie diffusément bornée de $C(K), Z$ est uniformément bornée sur le support de $F$.

Comme on l'a indiqué plus haut, on peut se ramener au cas où $F$ est vaguement dense, c'est-à-dire où $K$ est le support de $F$. Alors la quantité:

$$
\psi(\sigma)=\sup _{f \in Z}\left|\int f d \sigma\right|
$$

est une semi-norme s.c.i. sur l'espace de Banach $V$, donc majorée par un multiple de la norme. En particulier, il existe $M$ tel que

$$
\forall \sigma \in F \quad \forall f \in Z \quad\left|\int f d \sigma\right| \leqslant M .
$$

Et comme $F$ est dense dans $\mathscr{M}^{\mathrm{1}}(K)$, ceci est valable pour tout élément de $\mathscr{M}^{\mathrm{A}}(K)$, donc en particulier toute mesure de Dirac. Donc

\section{$\forall x \in K \forall f \in Z \quad|f(x)| \leqslant M$.}

On va maintenant prouver le théorème suivant:

THEOREME 7. Si la suite $\left(f_{n}\right)$ converge diffusément vers 0 , il existe une sous-suite de $\left(f_{n}\right)$ qui converge vers 0 en dehors d'une partie $F$-négligeable.

Nous aurons besoin, pour démontrer ce théorème, d'utiliser le lemme suivant:
LEMME 8. Soient $\left(f_{n}\right)$ une suite convergeant diffusément vers 0 et $\varepsilon>0$. Il existe une partie $M, F$-négligeable, de $K$ et une partie infinie $H$ de $N$ telles que

$$
\forall x \in K \backslash M \lim _{n \in H} \sup _{n \rightarrow \infty} f_{n}(x) \leqslant \varepsilon .
$$

Démonstration du lemme. Désignons par $O$ l'ensemble des couples $(U, H)$ formés d'un ouvert $U$ de $K$ et d'une partie infinie $H$ de $N$ tels que l'ensemble $\left\{x \in U \mid \lim \sup f_{n}(x)>\varepsilon\right\}$ soit $F$-négligeable, ordonné par la relation d'ordre:

$(U, H) \prec\left(U^{\prime}, H^{\prime}\right)$ si $\left[\left(U \subset U^{\prime}\right)\right.$ et $\left(H^{\prime} \backslash H\right.$ est fini) et $\left(H^{\prime}=H\right.$ où $\left.\left.U^{\prime} \neq U\right)\right]$.

Il va de soi que $O$ n'est pas vide puisque il contient $(\varnothing, N)$ et que l'énoncé du lemme est équivalent à l'exisfence d'un couple $(K, H)$ dans $O$. Nous allons prouver que $O$ est inductif, et qu'un élément maximal $(U, H)$ est nécessairement obtenu avec $U=K$.

Soit $\left(U_{i}, H_{i}\right)_{i \in I}$ une chaine dans $O$, et notons $V$ la réunion des $\left(U_{i}\right)_{i \in I}$. S'il existe un $i_{0} \in I$ tel que $V=U_{i_{0}},\left(U_{i_{0}}, H_{i_{0}}\right)$ est le plus grand élément de la chaîne. Sinon, il existe, en vertu du théorème de Lindelöf, une suite croissante $\left(i_{n}\right)$ telle que $V=\bigcup U_{i_{n}}$. On en conclut que $\left(i_{n}\right)$ est cofinal dans $I$. Il existe, d'après le lemme diagonal de Cantor, une partie infinie $H$ de $N$ telle que pour tout entier $n, H \backslash H_{i_{n}}$ soit fini.

Alors $(V, H)$ appartient à $O$ puisque $\mathcal{N}$ est un $\sigma$-idéal, et il majore tout élément de la chaîne.

Soit maintenant $(U, H)$ un élément maximal de $O$. Nous prouvons que l'hypothèse $K_{1}=K \backslash U \neq \varnothing$ amène une contradiction, et plus précisément, que l'ensemble des mesures $\sigma$ de $F \cap \mathscr{M}^{1}\left(K_{1}\right)$ telles que

$$
\limsup _{n \rightarrow \infty} \int f_{n} d \sigma \geqslant \varepsilon
$$

est un résiduel du compact $\mathscr{M}^{1}\left(K_{1}\right)$, contrairement à l'hypothèse de convergence diffuse de $\left(f_{n}\right)$ vers 0 .

Montrons d'abord que $F \cap \mathscr{M}^{1}\left(K_{1}\right)$, qui est un $G_{\delta}$ de $\mathscr{M}^{1}\left(K_{1}\right)$ est dense dans $\mathscr{M}^{1}\left(K_{1}\right)$. En effet, $F \cap \mathscr{M}^{1}\left(K_{1}\right)$ est une face de $\mathscr{M}^{1}\left(K_{1}\right)$ et son adhérence est une face fermée, c'est-à-dire l'ensemble des probabilités portées par un fermé $T$ de $K_{1}$. Si $F \cap \mathscr{M}^{1}\left(K_{1}\right)$ n'était pas dense dans $\mathscr{M}^{1}\left(K_{1}\right), T$ serait distinct de $K_{1}$, et l'ouvert $K_{1} \backslash T$ de $K_{1}$ serait $F$-négligeable. Le couple $(K \backslash T, H)$ majorerait alors strictement $(U, H)=\left(K-K_{1}, H\right)$ dans $O$, contrairement à la maximalité de $(U, H)$.

Pour prouver que l'ensemble $\left\{\sigma \in \mathscr{M}^{1}\left(K_{1}\right)|\limsup | \int f_{n} d \sigma \mid \geqslant \varepsilon\right\}$ est un résiduel, il suffit de montrer que, pour tout entier $p$, l'ouvert vague

$$
W_{p}=\bigcup_{n \geqslant p}\left\{\sigma \in \mathscr{M}^{1}\left(K_{1}\right) \mid \int f_{p} d \sigma>\varepsilon\right\}
$$

est partout dense dans $\mathscr{M}^{1}\left(K_{1}\right)$. Soit donc $W$ un ouvert vague non vide de $\mathscr{M}^{1}\left(K_{1}\right)$ Il existe dans $W$ une mesure discrète $\tau=\sum_{i=1}^{m} \alpha_{i} \varepsilon_{x_{i}}$ avec $\alpha_{i} \geqslant 0$ et $\sum_{1}^{m} \alpha_{i}=1$. 
$\mathrm{Il}$ existe alors des voisinages $V_{1}, \ldots, V_{m}$ de $x_{1}, \ldots, x_{m}$ dans $K_{1}$ tels que

$$
\left(y_{1}, \ldots, y_{m}\right) \in V_{1} \times V_{2} \times \ldots \times V_{m} \Rightarrow \sum_{i=1}^{m} \alpha_{i} \varepsilon_{y_{t}} \in W .
$$

L'hypothèse de maximalité de $(U, H)$ entraîne que pour tout ouvert non vide $V$ de $K_{1}$ et toute partie infinie $H^{\prime}$ de $H,\left(U \cup V, H^{\prime}\right) \notin O$, donc qu'il existe un point $y$ de $V$ tel que

$$
\lim _{n \in H^{\prime}} \sup _{n \rightarrow \infty} f_{n}(y)>\varepsilon
$$

donc aussi une partie infinie $H_{1}$ de $H^{\prime}$ telle que

$$
\forall n \in H_{1} \quad f_{n}(y)>\varepsilon \text {. }
$$

On peut donc construire inductivement des points $y_{1}, \ldots, y_{m}$ dans $V_{1}, \ldots, V_{m}$, et des parties infinies $H_{1}, \ldots, H_{m}$ de $H$ tels que

$$
\begin{gathered}
H_{m} \subset H_{m-1} \subset \ldots \subset H_{1} \subset H, \\
\forall n \in H_{i} \quad f_{n}\left(y_{i}\right)>\varepsilon .
\end{gathered}
$$

Pour ces points $y_{1}, \ldots, y_{m}$, et un entier $n \geqslant p$ choisi dans $H_{m}$, on a donc

$$
\begin{gathered}
\sigma=\sum_{1}^{m} \alpha_{i} \varepsilon_{y_{i}} \in W, \\
\int f_{n} d \sigma>\sum_{1}^{m} \alpha_{i} \varepsilon=\varepsilon,
\end{gathered}
$$

c'esț-à-dire $\sigma \in W \cap W_{p}$.

Ceci montre que $W_{p}$ est partout dense dans $\mathscr{M}^{1}\left(K_{1}\right)$, donc que $\bigcap_{p} W_{p}$ est un résiduel de $\mathscr{M}^{1}\left(K_{1}\right)$, de même que $F \cap\left(\bigcap_{p} W_{p}\right)$, ce qui achève la démonstration du lemme.

Démonstration du théorème 7. En appliquant le lemme précédent alternativement à la suite $\left(f_{n}\right)$ et à la suite $\left(-f_{n}\right)$, on peut trouver une suite décroissante $\left(H_{k}\right)$ de parties infinies de $N$ et une suite $M_{k}$ de boréliens $F$-négligeables telles que

$$
\forall x \in K \backslash M_{k} \lim _{n \in H_{k}} \sup _{n \rightarrow \infty}(-1)^{k} f_{n}(x) \leqslant 2^{-k} .
$$

Alors $M=\bigcup_{k} M_{k}$ est un borélien $F$-négligeable. Par ailleurs, en vertu du lemme diagonal de Cantor, il existe une partie infinie $H$ de $N$ telle que pour tout entiè $k$, $H \backslash H_{k}$ soit fini. On vérifie alors que

$$
\forall x \in K \backslash M \lim _{n \in H, n \rightarrow \infty} f_{n}(x)=0 .
$$

THEOREMe 9. Pour qu'une suite $\left(f_{n}\right)$ de fonctions continues sur $K$ converge diffusément vers 0 , il faut et il suffit qu'elle soit uniformément bornée sur le support de $F$ et que toute sous-suite de $\left(f_{n}\right)$ contienne une sous-suite qui converge $F$-presque partout vers 0 .

La partie directe du théorème résulte de la proposition 6 et du théorème 7 appliqué aux sous-suites de $\left(f_{n}\right)$. Inversement, si $\left(f_{n}\right)$ ne converge pas diffusément vers 0 , il existe $\sigma \in F$ et $\varepsilon>0$ tels que

$$
\limsup _{n \rightarrow \infty}\left|\int f_{n} d \sigma\right|>\varepsilon
$$

Si on pose alors $H=\left\{n \in N|| \int f_{n} d \sigma \mid>\varepsilon\right\}, H$ est infini. Si une sous-suite $\left(f_{n}\right)_{n \in H^{\prime}}$ de la suite $\left(f_{n}\right)_{n \in H}$ convergeait $F$-presque partout, elle convergerait $\sigma$-presque partout, et si la suite $\left(f_{n}\right)$ est uniformément bornée sur le support de $F$, elle est uniformément bornée sur le support de $\sigma$. Il résulterait alors du théorème de Lebesgue que $\lim _{n} \int f_{n} d \sigma=0$ alors que $\left|\int f_{n} d \sigma\right|>\varepsilon$ pour tout $n$ de $H^{\prime}$.

Corollarre 10. Si $\left(f_{n}\right)$ et $\left(g_{n}\right)$ sont deux suites de fonctions continues sur $K$; si, pour tout $n,\left|g_{n}\right| \leqslant\left|f_{n}\right|$ et si $\left(f_{n}\right)$ converge diffusément vers 0 , il en est de même de $\left(g_{n}\right)$.

Ceci résulte immédiatement du théorème précédent.

On s'intéresse maintenant aux suites de Cauchy pour la topologie diffuse. La proposition 6 montre qu'une telle suite est nécessairement uniformément bornée sur le support de $F$. De plus, si $\left(f_{n}\right)$ est une telle suite, on peut définir sur $F$ une fonction $\varphi$ par:

$$
\forall \sigma \in F \quad \varphi(\sigma)=\lim \int f_{n} d \sigma
$$

qui est définie puisque pour tout $\sigma$ la suite numérique de Cauchy $\left(\int f_{n} d \sigma\right)$ est convergente. On constate alors que $\varphi$ est de première classe sur $F$, et affine.

THEOREME 11. Soit $\varphi$ une fonction affine de première classe de Baire sur $F$. $\dot{I l}$ existe alors une fonction bornée $h$ sur $K$ de première classe telle que

$$
\forall \sigma \in F \quad \varphi(\sigma)=\int_{K} h d \sigma .
$$

Un théorème de $\mathrm{G}$. Mokobodzki [1] affirme sous des conditions moins restrictives sur $F$ l'existence d'une fonction borélienne $h$ telle que $\varphi(\sigma)=\int h d \sigma$, mais sans pouvoir préciser la classe de Baire de $h$. C'est pourquoi nous donnons une démonstration de ce théorème. Nous avons besoin du résultat intermédiaire suivant. , LEMME 12. Soient $\varphi$ une fonction affine de première classe sur $F$ et $\varepsilon>0$. Il existe alors une fonction de première classe $h_{\varepsilon}$ sur $K$ telle quie

$$
\forall \sigma \in F\left|\varphi(\sigma)-\int h_{\varepsilon} d \sigma\right| \leqslant \varepsilon .
$$

Soit $O$ l'ensemble des ouverts $U$ de $K$ sur lesquels existe une fonction de première classe $g$ vérifiant

$$
\forall \sigma \in F \cap \mathscr{M}^{1}(U) \quad\left|\varphi(\sigma)-\int g d \sigma\right| \leqslant \varepsilon .
$$


Soit $U$ la réunion de tous les éléments de $O$. Il existe une suite $\left(U_{n}\right)$ d'éléments de $O$ de réunion $U$, une suite $\left(g_{n}\right)$ de fonctions de première classe définies sur les $U_{n}$ telles que

$$
\forall \sigma \in F \cap \mathscr{M}^{1}\left(U_{n}\right)\left|\varphi(\sigma)-\int g_{n} d \sigma\right| \leqslant \varepsilon .
$$

Soit $\psi_{n}$ une partition de l'unité localement finie sur $U$ subordonnée au recouvrement $\left(U_{n}\right)$, et posons, pour $\sigma \in F \cap \mathscr{M}^{1}(U)$.

$$
\begin{aligned}
g & =\sum_{n=0}^{\infty} \psi_{n} g_{n}, \\
\alpha_{n} & =\int \psi_{n} d \sigma, \\
\sigma_{n} & =\left(1 / \alpha_{n}\right) \psi_{n} \sigma .
\end{aligned}
$$

La fonction $g$ est de première classe sur $U$, les $\left(\alpha_{n}\right)$ sont positifs et de somme 1, les $\sigma_{n}$ sont dans $F \cap \mathscr{M}^{1}\left(U_{n}\right)$ puisque $F$ est une face de $\mathscr{M}^{1}(K)$, et

$$
\sigma=\sum_{n=0}^{\infty} \alpha_{n} \sigma_{n}
$$

La fonction $\varphi$ se prolonge en une fonction linéaire $\tilde{\varphi}$ sur $V$, l'espace engendré par $F$, de première classe pour la norme puisque

$$
\tilde{\varphi}(\tau)=\left\|\tau^{+}\right\| \varphi\left(\frac{\tau^{+}}{\left\|\tau^{+}\right\|}\right)-\left\|\tau^{-}\right\| \varphi\left(\frac{\tau^{-}}{\left\|\tau^{-}\right\|}\right) .
$$

Puisque $V$ est un espace de Banach, $\tilde{\varphi}$ est continue donc bornée sur $F$, et dénombrablement additive. On a donc

$$
\varphi(\sigma)=\sum_{0} \alpha_{n} \varphi\left(\sigma_{n}\right)
$$

et

$$
\begin{aligned}
\left|\varphi(\sigma)-\int g d \sigma\right| & =\left|\sum_{0}^{\infty} \alpha_{n} \varphi\left(\sigma_{n}\right)-\sum_{0}^{\infty} \alpha_{n} \int g_{n} d \sigma_{n}\right| \\
& \leqslant \sum_{0}^{\infty} \alpha_{n}\left|\varphi\left(\sigma_{n}\right)-\int g_{n} d \sigma_{n}\right| \leqslant \sum_{0} \alpha_{n} \varepsilon \\
& \leqslant \varepsilon
\end{aligned}
$$

ce qui prouve que $U$ appartient à $O$, dont il est le plus grand élément.

Pour prouver le lemme, il faut montrer que $U=K$. Si $K_{1}=K \backslash U \neq \varnothing$, il existe une mesure $\sigma$ de $F$ telle que $\sigma\left(K_{1}\right) \neq \varnothing$; sinon la fonction $h_{a}$ obtenue en prolongeant $g$ par 0 sur $K_{1}$ est de première classe et satisfait les conditions du lemme. La mesure $\sigma^{\prime}=\sigma \mid K_{1} / \sigma\left(K_{1}\right)$ appartient alors à $F \cap \mathscr{M}^{1}\left(K_{1}\right)$ puisque $F$ est une face, et ceci prouve que $F \cap \mathscr{M}^{1}\left(K_{1}\right)$ est un fermé non vide de $F$. La restriction à ce fermé de $\varphi$ possède un point de continuité $\tau$.
Soient donc $\eta>0, \varrho_{1}, \ldots, \varrho_{m} \in C(K)$ tels que en désignant par $\Omega$ l'ouvert vague défini par

on ait

$$
\begin{aligned}
& \left\{\sigma|| \int \varrho_{j} d \sigma-\int \varrho_{j} d \tau \mid<\eta, j=1,2, \ldots, m\right\} \\
& \sigma \in \Omega \cap F \cap \mathscr{M}^{1}\left(K_{1}\right) \Rightarrow|\varphi(\sigma)-\varphi(\tau)|<\frac{1}{2} \varepsilon .
\end{aligned}
$$

En utilisant l'uniforme continuité des $\varrho_{j}$, il existe un recouvrement ouvert fini $\left(W_{1}, \ldots, W_{k}\right)$ de $K$ et une partition de l'unité $\left(\psi_{i}\right)$ subordonnée à ce recouvrement tels que, pour tout $i$,

Posons

$$
\left(x \in W_{i}, y \in W_{i}\right) \Rightarrow \sup _{j}\left|\varrho_{j}(x)-\varrho_{j}(y)\right|<\eta
$$

$$
\begin{aligned}
\alpha_{i} & =\int \psi_{i} d \tau \\
\tau_{i} & =\frac{1}{\alpha_{i}} \psi_{i} \tau \in F \cap \mathscr{M}^{1}\left(W_{i} \cap K_{1}\right) .
\end{aligned}
$$

Les $\alpha_{i}$ sont positifs de somme 1 , et $\tau=\sum_{i=1}^{k} \alpha_{i} \tau_{i}$. Si on définit

$$
\delta_{i}=\sup \left\{\varphi(\sigma)-\varphi\left(\sigma^{\prime}\right) \mid \sigma, \sigma^{\prime} \in F \cap \mathscr{M}^{1}\left(W_{i} \cap K_{1}\right)\right\}
$$

on voit, en utilisant que $\varphi$ est affine et que

$$
\left(\forall i=1,2, \ldots, k, \sigma_{i} \in F \cap \mathscr{M}^{1}\left(W_{i} \cap K_{1}\right)\right) \Rightarrow\left(\sum \alpha_{i} \sigma_{i} \in \Omega\right),
$$

l'on obtient

(1) $\quad \sum_{1}^{k} \alpha_{i} \delta_{i} \leqslant \sup \left\{\varphi(\sigma)-\varphi\left(\sigma^{\prime}\right) \mid \sigma, \sigma^{\prime} \in \Omega \cap F \cap \mathscr{M}^{1}\left(K_{1}\right)\right\} \leqslant \frac{1}{2} \varepsilon+\frac{1}{2} \varepsilon$.

Cette inégalité prouve l'existence d'un indice $p$ tel que $\alpha_{p}>0$ et $\delta_{p} \leqslant \varepsilon$.

Si l'on prolonge la fonction $g$ définie sur $U$ par la constante $\varphi\left(\tau_{p}\right)$ sur $W_{p} \cap K_{1}$, on obtient une fonction $g^{\prime}$ de première classe sur $U^{\prime}=U \cup W_{p} \neq U$ car $\alpha_{p}=\int \psi_{p} d \tau>0$. On a, pour toute mesure $\sigma$ de $F \cap \mathscr{M}^{1}\left(W_{p} \cap K_{1}\right)$

$$
\left|\varphi(\sigma)-\int g^{\prime} d \sigma\right|=\left|\varphi(\sigma)-\varphi\left(\tau_{p}\right)\right| \leqslant \delta_{p} \leqslant \varepsilon
$$

d'où, puisque $F$ est une face, et que $\varphi$ est affine

$$
\forall \sigma \in F \cap \mathscr{M}^{1}\left(U^{\prime}\right) \quad\left|\varphi(\sigma)-\int g^{\prime} d \sigma\right| \leqslant \varepsilon .
$$

Ceci prouve que $U$ n'était pas le plus grand élément de $O$, donc que l'hypothèse $K_{1} \neq \varnothing$ était absurde. Le lemme est donc prouvé.

Démonstration du théorème 11. Soit $\varphi$ une fonction affine de première classe sur $F$. Il existe d'après le lemme précédent une suite $\left(g_{n}\right)$ de fonctions de première classe $g_{n}$ telles que $\forall n \in N \forall \sigma \in F \quad\left|\varphi(\sigma)-\int g_{n} d \sigma\right| \leqslant 2^{-n}$. 
Il en résulte que

$$
\forall \sigma \in F \quad\left|\int\left(g_{n+1}-g_{n}\right) d \sigma\right|<2^{1-n} .
$$

Si l'ensemble $A_{n}=\left\{x \in K||\left(g_{n+1}-g_{n}\right)(x) \mid>2^{1-n}\right\}$ n'était pas $F$-négligeable, il existerait $\sigma \in F$ tel que $\sigma\left(A_{n}\right)>0$, donc $\tau \leqslant \sigma, \tau \neq 0$ tel que $g_{n+1}-g_{n}>2^{1-n} \tau$-presque partout (ou $g_{n}-g_{n+1}>2^{1-n}$ ). Alors $\frac{\tau}{\|\tau\|} \in F$ et

$$
\left|\int\left(g_{n+1}-g_{n}\right) d \frac{\tau}{\|\tau\|}\right|>2^{1-n},
$$

contrairement à ce qui précéde. On définit alors

$$
\begin{aligned}
h_{0} & =g_{0}, \\
\forall n \geqslant 0, \quad h_{n+1} & =\sup \left(-2^{1-n}, \inf \left(2^{2-n}, g_{n+1}-g_{n}\right)\right) .
\end{aligned}
$$

La série $h_{n}$ converge uniformément vers une fonction de première classe $h$, et puisque

$$
\dot{\forall} \in \mathbb{\forall} \forall \sigma \in F \quad \int h_{n+1} d \sigma=\int\left(g_{n+1}-g_{n}\right) d \sigma
$$

$$
\int h d \sigma=\lim _{n \rightarrow \infty} \int g_{n} d \sigma=\varphi(\sigma)
$$

ce qui achève la démonstration.

COROLlaRe 13. Si $\left(f_{n}\right)$ est une suite de Cauchy pour la topologie diffuse, il existe une suite bornée $\left(g_{n}\right)$ de fonctions continues sur $K$ qui converge simplement sur $K$ et telle que la suite $\left(f_{n}-g_{n}\right)$ converge diffusément vers 0 .

Il résulte de ce qui précéde qu'il existe une fonction de première classe $h$ sur $K$ telle que:

$$
\forall \sigma \in F \quad \int f_{n} d \sigma \rightarrow \int h d \sigma .
$$

$\mathrm{Si}\left(g_{n}\right)$ est une suite bornée de fonctions continues sur $K$ qui converge simplement vers $h$, on aura

$$
\forall \sigma \in F \quad \int\left(f_{n}-g_{n}\right) d \sigma \rightarrow \int h d \sigma-\int h d \sigma=0
$$

ce qui termine la démonstration.

THEOREMB 14. Pour qu'une suite $\left(f_{n}\right)$ de fonctions continues sur $K$ soit une suite de Cauchy pour la topologie diffuse, il faut et il suffit qu'elle soit bornée uniformément sur le support de $F$ et qu'il existe une fonction h de première classe sur $K$ telle que toute sous-suite de $\left(f_{n}\right)$ contienne une sous-suite convergeant $F$-presque partout vers $h$.

Ceci résulte immédiatement du corollaire 13 et du théorème 11.

Proposition 15. Si $K$ est un compact parfait et $F$ l'ensemble des probabilités diffuses, si $\left(f_{n}\right)$ est the suite de Cauchy pour la topologie diffuse, $\left(f_{n}\right)$ possède une sous-suite qui converge simplement partout.
Il résulte de la proposition 6 que $\left(f_{n}\right)$ est uniformément bornée, et du théorème 14 que $\left(f_{n}\right)$ possède une sous-suite qui converge simplement $F$-presque partout, c'està-dire sur le complémentaire d'un ensemble dénombrable $D$. Par utilisation du lemme diagonal, on peut encore extraire de cette sous-suite une suite qui converge en chaque point de $D$, donc en tout point de $K$.

Remarque 16 . On ne peut en général renforcer le théorème 7 en affirmant que le suite $\left(f_{n}\right)$ elle-même converge $F$-presque partout vers 0 .

Prenons $K=[0,1], F$ l'ensemble des mesures diffuses sur $[0,1]$. Pour tout entier $n$ il existe $p$ et $q$ entiers tels que

$$
n=2^{p}+q, \quad 0 \leqslant q<2^{p} .
$$

Soient $I_{n}$ l'intervalle $\left[\frac{q}{2^{p}}, \frac{q+1}{2^{p}}\right]$ et $f_{n}$ une fonction continue sur $K$, à valeurs dans $[0,1]$, valant 1 sur $I_{n}$ et 0 hors de $\left[\frac{q-1}{2^{p}}, \frac{q+2}{2^{p}}\right]$.

Alors $\limsup _{n \rightarrow \infty} f_{n}=1$. Néanmoins, si $\sigma$ est une mesure diffuse, la fonction $x \rightarrow \sigma([0, x])$ est continue, donc uniformément continue; il existe donc pour tout $\varepsilon>0$ un $\eta>0$ tel que $\operatorname{diam} A<\eta$ entraîne $\sigma(A)<\varepsilon$. Il en résulte que

$$
\int f_{n} d \sigma \rightarrow 0 \text {. }
$$

La suite $\left(f_{n}\right)$ converge donc diffusément vers 0 , mais ne converge vers 0 en aucun point.

Remarque 17. L'hypothèse que $F$ est une face $G_{\delta}$ ne peut être remplacée dans le théorème 7 par l'hypothèse que $F$ est une face $K_{\sigma}$.

Prenons encore $K=[0,1]$, mais pour $F$ l'ensemble des probabilités absolument continues par rapport à la mesure de Lebesgue, et à densité bornée. On sait que la suite $\left(f_{n}\right)$ définie par $f_{n}(t)=\sin n \pi t$ converge vers 0 pour $\sigma\left(L^{1}, L^{\infty}\right)$, donç pour la topologie diffuse qui en est alors la trace sur $C(K)$.

Néanmoins, si une sous-suite $\left(f_{n_{k}}\right)$ convergeait $F$-presque partout vers 0 , c'està-dire presque partout, on aurait, puisque

Or

$$
\begin{gathered}
\sup _{n, t}\left|f_{n}(t)\right|=1, \\
\lim _{k \rightarrow \infty} \int_{0}^{1} f_{n_{k}}^{2}(t) d t=0,
\end{gathered}
$$

$$
\forall n \geqslant 1 \quad \int f_{n}^{2}(t) d t=\frac{1}{2} .
$$

On en déduit qu'aucune sous-suite ne converge $F$-presque partout vers 0 , alors que $F$ est une face $K_{\sigma}$, dense dans $\mathscr{M}^{1}(K)$.

THEOREME 18. Dans les, théorèmes 9 et 14, on peut remplacer l'hypothèse que les $\left(f_{n}\right)$ sont continues par l'hypothèse que les $\left(f_{n}\right)$ sont boréliennes bornées, à condition 
de remplacer "suite bornée sur le support de F" par "suite uniformément bornée sur le complémentaire d'un borélien $F$-négligeable, et, dans l'énoncé 14 , "une fonction $h$ de première classe" par "une fonction h borélienne".

En effet, si les $\left(f_{n}\right)$ sont boréliennes sur $K$, il existe un espace polonais $Q$ et une bijection continue $\pi$ de $Q$ sur $K$ atels que les $\left(f_{n} \circ \pi\right)$ soient continues. Si les $\left(f_{n}\right)$ sont bornées, il existe une compactification métrisable $\hat{Q}$ de $Q$ telle que les $f_{n} \circ \pi$ se prolongent en fonctions continues $\hat{f}_{n}$ sur $\hat{Q}$.

L'ensemble $\hat{F}$ des probabilités sur $Q$ dont l'image par $\pi$ est dans $F$ est une face $G_{\delta}$ de $\mathscr{M}^{1}(Q)$, donc une face $G_{\delta}$ de $\mathscr{M}^{1}(\widehat{Q})$ puisque $\mathscr{M}^{1}(Q)$ est une face $G_{\delta}$ de $\mathscr{M}^{1}(\widehat{Q})$.

Il suffit alors d'appliquer les théorèmes 9 ou 14 aux $\hat{f}_{n}^{*}$ sur $\hat{Q}$, pour obtenir le résultat cherché, compte tenu de ce que $\hat{h} \circ \pi^{-1}$ est borélienne si $\hat{h}$ est de première classe sur $\hat{Q}$, l'application $\pi^{-1}$ étant borélienne.

\section{Reference}

[1] G. Mokobodzki, Sur la limite faible d'une suite de fonctions boréliennes, Séminaire de Théorie du Potentiel de Paris ${ }^{\circ} 2$, Lecture Notes in Math. $n^{\circ} 563$. 